Conclusion* Using a multimodal approach, including IHC and FACs, we demonstrate that the metastatic TME in HGSOC is significantly different to the primary TME. These findings provide an initial explanation as to why immune checkpoint inhibitors have failed in HGSOC and warrant further investigation.

\section{FERTILITY OUTCOMES FOLLOWING FERTILITY SPARING SURGERY FOR THE MANAGEMENT OF EARLY-STAGE CLEAR CELL OVARIAN CARCINOMA; A SYSTEMATIC REVIEW}

A Prodromidou*, C Theofanakis, N Thomakos, D Haidopoulos, A Rodolakis. st Department of Obstetrics and Gynecology, Medical School, National and Kapodistrian University of Athens, "Alexandra" Hospital, Athens, Greece

\subsection{6/ijgc-2021-ESG0.416}

Introduction/Background* The application of fertility sparing surgery (FSS) in patients with clear cell ovarian carcinoma (CCOC) has been extensively criticized, even in patients with stage IA or IC disease, due to the high reported recurrence rates and the resistance to chemotherapy. The objective of the present study was to evaluate the obstetric and fertility outcomes of patients with early stage CCOC following fertility sparing surgery (FSS).

Methodology Three electronic databases were systematically searched for articles published in the field up to December 2020 using the terms "ovarian cancer", "clear cell", "fertility sparing", "conservative treatment". Studies that reported pregnancy and obstetric outcomes after FSS for the management of early stage CCOC were considered eligible for inclusion.

Result(s)* A total of 5 retrospective studies with 60 patients with CCOC who underwent FSS were included. Mean patients' age was 34.8 years. The total clinical pregnancy rate was $32 \%$ with a proportion of $24 \%$ of live birth rates in 12 of the included patients. The median interval from disease management to pregnancy was 41.5 months. Recurrence rate was $16.6 \%$ among the included patients. Survival and recurrence rates were not different in patients who had FSS compared to those who had radical surgery.

Conclusion* Fertility-sparing treatment for stage IA/IC CCOC seems to be an acceptable treatment option for selected women of reproductive age with a strong desire of fertility preservation. Further larger multicenter studies and studies derived from registries are warranted to validate the special aspects of the procedure and to designate the potential candidates who will receive survival and fertility benefit from fertility-sparing surgery.

\section{SIGNIFICANT SURVIVAL BENEFIT IS ASSOCIATED WITH COMPLETE CYTOREDUCTION IN DELAYED CYTOREDUCTIVE SURGERY FOR ADVANCED OVARIAN CANCER}

V Cassar*, S Rundle, P Korompelis, A Fisher, A Kucukmetin, N Ratnavelu, C Ang. Queen Elizabeth Hospital Gateshead, Gynaecology Oncology, Gateshead, UK

\subsection{6/ijgc-2021-ESG0.417}

Introduction/Background* The current gold standard in the surgical management of advanced ovarian cancer(AOC) recommended by ESGO and ASCO is complete resection of all visible disease. If this is not deemed possible in the upfront setting, then interval cytoreductive surgery should be undertaken after $\leq 4$ cycles of NACT. Occasionally due to the persistence of unresectable sites of disease on interval scanning or because of factors associated with fitness for surgery, surgery in the interval setting may not be possible.

Limited published data assessing outcomes from surgery delayed to after 5 or 6 cycles of NACT(delayed cytoreductive surgery) suggests a potential benefit over no surgery and suggests that if interval cytoreductive surgery is not possible, then the clinician might consider delayed surgery on a case by case basis.

We sought to review the outcomes of patients with AOC presenting to the Northern Gynaecological Oncology Centre (NGOC) who underwent delayed surgery.

Methodology This study is a retrospective analysis looking at patients with AOC referred to the Northern Gynaecological Oncology Centre(NGOC), Gateshead, UK between 2017 and 2020, who were not deemed suitable to undergo either primary cytoreductive or interval debulking surgery and instead had DDS following 5 or 6cycles of NACT .

Result(s)* Over the 3 year period, 401 patients with AOC were referred to the NGOC MDT. 25 patients had delayed surgery, $16(66.7 \%)$ patients had surgery after 5 cycles of chemotherapy, $7(28 \%)$ patients had surgery after 6 cycles and there was incomplete data in 1 patient. The median age was 64. $66.7 \%(16 / 24)$ presented with stage IIIc disease, 12.5\%(3/ 24) were stage IVa and $20.8 \%(5 / 24)$ were stage IVb. The majority had high grade serous carcinoma(91.7\%). All patients had platinum based NACT(Carboplatin), 17 had dual agent chemotherapy with Paclitaxel and 3 had additional Bevacizumab.

18/19 received post-operative chemotherapy and complete cytoreduction was achieved in $17 / 24(71 \%)$ patients. Suboptimal cytoreduction was more common in patients having surgery after 6 cycles and the median overall survival was 24 months in those completely cytoreduced compared to 9.5 months in those with residual disease $(p=0.03)$.

Conclusion* A significant improvement in OS is seen in women who are completely cytoreduced after $\geq 5$ cycles compared to those with residual disease post-operatively. Therefore, if complete clearance can be achieved, cytoreductive surgery should be offered to all patients even after $\geq 5$ cycles.

\section{DIAGNOSTIC FEATURES OF OVARIAN CANCER IN PREMENOPAUSAL WOMEN}

${ }^{1}$ I Pelayo, ${ }^{1} \mathrm{~V}$ Corraliza-Galan*, ${ }^{1} \mathrm{C}$ Martin-Gromaz, ${ }^{1} \mathrm{MJ}$ Pablos-Antona, ${ }^{1} \mathrm{C}$ Del Valle-Rubido, ${ }^{1}$ E Cabezas-Lopez, ${ }^{1} \mathrm{D}$ Rubio-Marin, ${ }^{2} \mathrm{~B}$ Perez-Mies, ${ }^{1} \mathrm{~L}$ Abarca-Martinez, ${ }^{1} \mathrm{E}$ MoratallaBartolome, ${ }^{1} \mathrm{C}$ Sanchez-Martinez, 'I Lazaro de la Fuente. 'Ramon y Cajal Hospital, Obstetrics and Gynecology, Madrid, Spain; ${ }^{2}$ Ramon y Cajal Hospital , Pathology, Madrid, Spain

\subsection{6/ijgc-2021-ESG0.418}

Introduction/Background* Ovarian cancer (OC) is the most lethal gynaecological malignancy worldwide, specially because it's diagnosed as advanced-stage disease. Clinical aspects are inespecific and appear in advanced stages. Ultrasound study (US) remains the primary modality for assessment of ovarian tumors. Computed tomography (CT) imaging is the standard of care for pre-operative evaluation of ovarian cancer patients. Serum CA125 assay has low sensitivity in early stages. 